\title{
Covid-19: Why herd immunity was not approached anywhere? Ultrametric diffusion modelling of virus spread in hierarchically clustered population.
}

\author{
Andrei Khrennikov \\ Linnaeus University, International Center for Mathematical Modeling \\ in Physics and Cognitive Sciences Växjö, SE-351 95, Sweden
}

June 6, 2021

\begin{abstract}
In spite of numerous predictions, the natural herd immunity for covid-19 visru had not been approahed anywhere in the world. Thus, the traditional mathematical models of disease spread demonstrated their inability to describe adequately the covid-19 pandemic. In author's works, the novel model of the disease spread was developed. This model reflects the basic features of the covid-19 pandemic: a) the social clustering character of virus spread, b) . Social clustering is mathematically modelled with ultrametric spaces having the treelike geometry encoding hierarchy of the regulation constraints. The virus spread is described by ultrametric diffusion or random walk on the hierarchic energy landscape. In contrast to the standard models which are characterized by the exponential decrease of the probability to become infected - at the stage of approaching of the herd immunity, the ultrametric model is characterized by the power law. Moreover, the model gives the possibility to quantify the influence of restriction measures up to the lockdown. Our main result is that the play with restrictions, including lockdowns, is counterproductive and leads to the essential slowdown of approaching the herd immunity or even makes this impossible.
\end{abstract}




\section{Introduction}

In this paper, we structure the presentation of the model suggested in $[1,2]$, make it clearer and shorter by eliminating the technical details.

The disease spread during the covid-19 pandemic is characterized by specialties which have to be taken into account in mathematical modeling. It seems that the majority of the standard mathematical models [3]-[7] do not take these specialties into account [8]-[14]. This implies their inadequacy to real virus behavior and, in particular, inability to predict the dynamics of approaching the herd immunity. ${ }^{1}$ In particular, in Sweden where approaching the herd immunity was the important component of the state strategy, incapacity of mathematical models to make adequate predictions lead to statements in mass media about usefulness of mathematical modeling in general. ${ }^{2}$

In author's works [1] (see also the joint paper with Oleschko [2]), a novel model of disease spread was suggested and elaborated. In particular, our model violates the basic assumption of "conventional disease spread modeling", namely, on homogeneous mixing of the population: "people make their contact at random and without restricting them only to a smaller subgroup". Of course, it is well known that this assumption is rarely justified - precisely because of the social clustering of population. Nevertheless, homogeneous mixing is a standard assumption to make the mathematics tractable. During the previous epidemics the validity of the conventional models was not questioned, because the state authorities did not impose the rigid and extensive measures of control over the population. Imposing such measures up to lockdowns made these models not completely inadequate to covid19 pandemic.

Our model reflects the basic features of the covid-19 pandemic (see section 3 for the details, discussion, and medical motivation):

- ASO the social clustering character of virus spread under restrictions imposed by the state authorities,

- $\mathbf{A S 1}$ the probability $p_{I}(C, t)$ to become infected in a social cluster $C$ at the moment $t$ is not proportional to the number $I(t)$ of infected people.

In particular, under assumption AS1 the basic reproduction number $R_{0}$ (see section 2) is not the critical parameter of the model determining whether the infection spreads or vanishes.

\footnotetext{
${ }^{1}$ We consider immunity which is combination of innate and adaptive components and not coupled to mass-vaccination.

${ }^{2}$ Models of Swedish mathematicians sequentially "predicted" approaching the herd immunity in April, May, June 2020 [10]-[12]. Then they gave up.
} 
Social clustering is mathematically modeled with ultrametric spaces (see also [15]-[19]) having the treelike geometry encoding the clustering hierarchy. The virus spread under assumption AS0+AS1 is described by ultrametric diffusion or random walk on the hierarchic energy landscape [20]-[23], [24]-[31].

In contrast to the standard models [3]-[7] which are characterized by the exponential decrease of the probability to become infected - at the stage of approaching the herd immunity $\left(R_{0}<1\right)$, the ultrametric model is characterized by the power law [1]. Moreover, the model gives the possibility to quantify the influence of restriction measures up to the lockdown. The social restrictions, both traditional and imposed by the state authorities, are characterized by the parameter $\alpha>0$. And the later determines the power law for the dynamics of population's immunity.

$$
p_{\operatorname{Im}}(t) \sim 1-t^{-q}, q=1 / \alpha,
$$

for large $t$. If the barriers are high, $\alpha>>1$, then probability that an individual in population is immune increases very slowly. In short stronger restrictions imply less possibility to approach the herd immunity in realistic time. Thus, the play with restrictions, including lockdowns, is counterproductive and leads to the essential slowdown of approaching the herd immunity or even makes this impossible

For experts in probability theory, we remark that $p_{I}(C, t)$ is interpreted as the subjective probability, in the line with the methodology of decision making. It is crucial that $p_{I}(C, t)$ is the probability to become infected, not being infected (see section 4 for the discussion).

Finally, we note that the standard assumption on homogeneous mixing of the population was also turned down in the paper of Britton et al. [32] in which the role of population heterogeneity in spread of covid-19 was analyzed. The main difference with our approach is that in [32] the hierarchic structure of social clustering and hence the hierarchy of barriers between clusters is not taken into account; the real metric was used, instead of ultrametric. As usual, Britton et al. [32] proceeded under negation of the assumption AS1 (cf. [33]-[35]). This modeling did not imply the power law (1).

\section{The conventional models and basic reproduction number}

One of the basic parameters of the conventional models [3]-[7] is the basic reproduction number (denoted by $R_{0}$ ) quantifies transferability of a disease: the average number of individuals that a single infec- 
tious person will infect. This is the basic parameter that determines infection's spreading:

- $R_{0}>1$ : the infection spreads exponentially,

- $R_{0}<1$ : the infection vanishes exponentially,

- $R_{o}=1$ : the disease is endemic, i.e., it will move throughout the population but not increase or decrease.

For us it is interesting that whether the infection spreads or disappears depends on the single parameter and epidemic's behavior is always exponential; when $R_{0}$ becomes less than 1 , the herd immunity is approached very quickly.

As was noted, the basic reproduction number is not the critical parameter of our model. The regimes of infection's spreading and vanishing are not exponential. Instead of $R_{0}$, we consider the probability of infection's transition from one cluster to another, it depends smoothly on the parameter $\alpha$ determining the strength of the isolation measures (section 6, (12)).

\section{The basic assumptions beyond the mathematical model}

The readers who are mainly interested in mathematical modeling and its output can directly jump to the section 12 .

The main epidemiological assumption beyond our ultrametric model is AS0: virus' spread in population is constrained by the hierarchic social cluster structure and it "respects" this structure. Assumption AS1 is partially a consequence of AS0, but we also present some biologic and epidemic reasons in its support.

- Covid in air. The virus is not dangerous at the open air, especially if people follow the recommendation to keep 1,5 m distance between them.

- Asymptomatic individuals. As was recently announced [36], WOH collected a lot of statistical data showing that asymptomatic individuals transmit covid-19 virus to other people with very low probability.US Centers for Disease Control and Prevention estimates that about a third of coronavirus infections (35\%) are asymptomatic [37]. Hence, about $35 \%$ of infected people practically do not contribute in disease spread.

- No mass-events. Another important restriction supporting AS1 is that even in Sweden, mass-events were forbidden, so no public concerts, neither football matches. 
- Superspreaders. Spread of coronavirus has the following feature - the presence of superspreaders of infection. One person can infect really many people. Thus, single person's contribution in disease spread can be essentially higher than contribution of a few hundreds of usual asymptomatic individuals or many presymptomatic individuals. ${ }^{3}$

In April-May 2020, for Swedish population we could assume that

AS2 The number of susceptible people $S(t)$ is so large comparing with the number $I(t)$ of those who are infected or the number $R(t)$ of recovered that we can consider it as constant, $S(t)=$ const, and exclude it from model's dynamical equations.

This assumption implies that, for an individual in population under consideration, the probability to become infected practically does not depend on the number of recovered. The population is rather far from approaching herd immunity and a disease spreader is surrounded (with the high degree of approximation) by susceptible people. Thus the number of recovered people $R$ also can be excluded from dynamics. ${ }^{4}$

Denote the probability, for a person from social cluster $C$, to become infected at the instance of time $t$ by the symbol $p_{I}(C, t)$ (see section 4 for interpretation of this probability). To write the evolution equation for probability $p_{I}(C, t)$, we impose the additional assumption:

AS3 The distribution of social clusters in the society is uniform: all clusters represented by balls of the same radius have the same measure that is equal to balls' radius.

Mathematically AS3 is formalized through the use of the Haar measure $\mu$ on $\mathbf{Q}_{p}$. We understand that this is a strong restriction on the

\footnotetext{
${ }^{3}$ Superspreader is an unusually contagious individual who has been infected with disease; someone who infected the number of people far exceeding the two to three. As was pointed out in MIT Technology Review [38]: "For covid-19, this means $80 \%$ of new transmissions are caused by fewer than $20 \%$ of the carriers - the vast majority of people infect very few others or none at all, and it is a select minority of individuals who are aggressively spreading the virus. A recent preprint looking at transmission in Hong Kong supports those figures, while another looking at transmission in Shenzhen, China, pegs the numbers closer to $80 / 10$. Lots of outbreaks around the world have been linked to single events where a superspreader likely infected dozens of people. For example, a choir practice in Washington State infected about 52 people; a megachurch in Seoul was linked to the majority of initial infections in South Korea; and a wedding in Jordan with about 350 guests led to 76 confirmed infections." The bad news is that, for the moment, we cannot identify diagnostically superspreaders.

${ }^{4}$ One of specialties of covid-19 pandemic in Sweden in spring 2020 was that people did not make tests (and testing without symptoms was not recommended by the state epidemic department). The number of infected was very low, because the presence of the essential component of asymptomatically infected people.
} 
social structure of society. But, the main reason for its imposing is just simplification of mathematics. We can consider other distributions on $\mathbf{Q}_{p}$ assigning different weights to social clusters represented by balls of the same radius. (We recall that any point of a ball can serve as its center.)

\section{Probability to become infected and its dynamics}

We consider socially clustered population (see section 5 for mathematical formalization and discussion). The quantity $p_{I}(C, t)$, the probability that a person belonging to social cluster $C$ can become infected at the instant of time $t$, is the basic quantity of our model. We point to the difference from the standard SIR-like models: $p_{I}(C, t)$ is not the proportion of infected people in cluster $C$ at the instant of time $t$, i.e.,

$$
p_{I}(C, t) \neq \frac{N_{I}(C, t)}{N},
$$

where $N$ is the number of people in $C$ and $N_{I}(C, t)$ is the number of infected people in $C$ at the instant of time $t$. Thus, $p_{I}(C, t)$ has no straightforward relation to the number of infected people $N_{I}(C, t)$.

Probability $p_{I}(C, t)$ is determined by context $C(C, t)$, the complex of social, economic, and epidemiological conditions in cluster $C$ at the instant of time $t$, i.e., this is contextual probability. ${ }^{5}$

One of the possibilities to interpret the probability to become infected in context $C(C, t)$ is to use the subjective interpretation of probability. This interpretation became popular even in quantum physics, under the name of Quantum Bayesianism (QBism) [62]. By this interpretation $p_{I}(C, t)$ is subjective probability that is assigned by decision maker to the event that an individual in the social cluster $C$ would become infected. We stress that a social cluster is a domain in social space, so it need not be determined simply by geography (although geographic location place the important role in determination of $C$ ).

\footnotetext{
${ }^{5}$ Such probabilities are considered, e.g., in quantum theory [39], where it is difficult, if possible at all, to introduce "hidden variables" determining probabilities. We remark that the situation in epidemiology, especially with respect to the covid-19 epidemic, is similar to quantum physics and more general quantum-like modeling in cognition, psychology, and decision making. It is impossible to determine "hidden variables" behind many events. People and social clusters of people reacts to covid-19 in very different ways: there are bio-medical, social, and may be even psychological hidden variables. Context $C(C, t)$ determines their distribution, but it seems to be impossible to find these probability distributions of "hidden variables" and their dependence on contexts.
} 
Subjective probability is widely used in decision making as a part of subjective utility theory.

The problem under consideration is by knowing probabilities to become infected in social clusters $C_{i}, i=1, \ldots, M$, at time $t_{0}, p_{I}\left(C_{i}, t_{0}\right)$, to estimate the probability for cluster $C_{k}$ at later instances of time $t>t_{0}, p_{I}\left(C_{k}, t\right)$.

Dynamics $t \rightarrow p_{I}\left(C_{k}, t\right)$ is described by the master equation. To write this equation, we have to consider conditional probabilities (also known as transition probabilities):

$p\left(C_{k} \mid C_{i} ; t\right)$ is the probability, for a person in social cluster $C_{k}$, to get infection from a person from cluster $C_{i}$. These conditional probabilities represent intensities to become infected.

The master equation has the form:

$$
\frac{d}{d t} p_{I}\left(C_{k}, t\right)=\sum_{i \neq k}\left[p\left(C_{k} \mid C_{i} ; t\right) p_{I}\left(C_{i}, t\right)-p\left(C_{i} \mid C_{k} ; t\right) p_{I}\left(C_{k}, t\right)\right] .
$$

By using infinitesimals, we can write this probability balance equation in the form:

$p_{I}\left(C_{k}, t+d t\right)=p_{I}\left(C_{k}\right)+\sum_{i \neq k}\left[p\left(C_{k} \mid C_{i} ; t\right) p_{I}\left(C_{i}, t\right)-p\left(C_{i} \mid C_{k} ; t\right) p_{I}\left(C_{k}, t\right)\right] d t$.

The term $p\left(C_{k} \mid C_{i} ; t\right) p_{I}\left(C_{i}, t\right)$ gives the intensity of transition of infection from cluster $C_{i}$ to cluster $C_{k}$. The probability to become infected in cluster $C_{k}$ increases due to transfer of infection from other clusters. Thus meaning of the positive term in the right-hand side of (4) is clear.

The negative term describes decreasing of infection in cluster $C_{k}$ resulting from the "flow of infection" from $C_{k}$ to other clusters. This flow generates decrease of the probability to become infected in $C_{k}$. To describe the latter process, we should consider disease spreaders and their transitions between social clusters, say from Bronx to Manhattan and vice verse. The quantity $p\left(C_{k} \mid C_{i} ; t\right) p_{I}\left(C_{i}, t\right)$ gives the probability that somebody from social cluster $C_{k}$ (Manhattan) would be infected by somebody who comes to $C_{k}$ from $C_{j}$ (say from Bronx). But at the same time some infected people from Manhattan, $C_{k}$ can go to Bronx, $C_{i}$, and infect people here. By "being busy with infecting people in Bronx", people from Manhattan cannot infect people in their own social cluster, so the probability to become infected in Manhattan decreases by $p\left(C_{i} \mid C_{k} ; t\right) p_{I}\left(C_{k}, t\right) d t$. 


\section{$5 \quad$ Hierarchic treelike geometry of so- cial clusters}

We represent the human society as a system of hierarchically coupled disjoint clusters. Theory of ultrametric spaces is one of the basic mathematical tools for representation of such clusters purpose. Geometrically ultrametric spaces can be represented as trees with hierarchic levels. Ultrametricity means that this metric satisfies so-called strong triangle inequality:

$$
\rho(x, y) \leq \max \{\rho(x, y), \rho(y, z)\},
$$

for any triple of points $x, y, z$. Define balls as usual in metric spaces $B_{R}(a)=\left\{x: \rho_{p}(x, a) \leq R\right\}$, where $a$ is a center of the ball and $R>0$, is its radius. We use the ultrametric balls to represent mathematically social clusters, any cluster is slit into disjoint sub-clusters, each of them is split into its own (disjoint) sub-clusters and so on. Inclusion relation generates the hierarchy on the set of social clusters.

In the works of the author and his collaborators [15]-[19], ultrametric spaces (geometrically hierarchic trees) were applied for modeling of cognitive, psychological, and social phenomena. This modeling was based on invention of systems of discrete social (or mental in cognitive studies) coordinates $x=\left(x_{m}\right)$ characterizing (psycho-)social states of individuals. The treelike representation of social states is based on selection of hierarchically ordered social factors enumerated by index $m \in \mathbf{Z}=\{0, \pm 1, \pm 2, \ldots\}$. The social importance of coordinates $x_{m}$ decreases with increase of $m$ and increases with decrease of $m$; e.g., social coordinate $x_{0}$ is more important than any $x_{j}, j>0$, but it less important than any $x_{j}, j<0$. The coordinate $x_{0}$ can be considered as a reference point. Depending on context (say socio-economic or socio-epidemic), it can be shifted to the right or to the left. Therefore it is convenient to use positive and negative indexes determining two different directions of social importance of coordinates.

We consider discrete social coordinates, generally, for each $m$, there $N_{m}$ possible values, $x_{m}=0,1, \ldots, N_{m}-1$, and $N_{m}$ can vary essentially with $m$. In the treelike representation, numbers $N_{m}$ determine the number of branches leaving vertexes. Analysis on such trees is mathematically complicated $[28,29]$ and we restrict modeling to homogeneous trees for that $N_{m}$ does not depend on $m$. Moreover, by the pure mathematical reasons it is convenient to select $N_{m}=p$, where $p>1$ is the fixed prime number.

Thus, a social state $x$ is represented by a vector of the form:

$$
x=\left(x_{-n}, \ldots, x_{-1}, x_{0}, x_{1}, \ldots, x_{m}\right), x_{j} \in\{0,1, \ldots, p-1\} .
$$


The vector representation of psychical, mental, and social states is very common in psychology and sociology. The essence of our approach [15]-[18] is the hierarchic ordering of coordinates leading to introduction of ultrametric on the state space, see (??).

For our purpose, modeling of epidemic, we can consider, for example, the following hierarchic system of social coordinates; for simplicity, let index $m=0,1,2, \ldots$, so the coordinate $x_{0}$ is the most important. It is natural to use it to denote states (e.g., Sweden, Russia, USA,...); then, for example, $x_{1}$ can be used for age; $x_{2}$ for chronic diseases, $x_{3}$ gender, $x_{4}$ for race, $x_{5}$ for the town of location, $x_{6}$ for the district, $x_{7}$ for profession, $x_{8}$ for the level of social activity, $x_{9}$ for the number of children, and so on. We understand that such ranking of the basic social factors related to the covid-19 epidemic is incomplete. We also stress that hierarchy of social factors involved in the covid-19 epidemic can be selected depending on the state, i.e., for each state we create its own system of social clustering coupled to this epidemic.

It is convenient to proceed with variable number of coordinates, i.e., not fix $n$ and $m$. This gives the possibility to add new coordinates. The space of such vectors can be represented by rational numbers of the form

$x=x_{-n} p^{-n}+\ldots+x_{-1} p^{-1}+x_{0}+x_{1} p+\ldots+x_{m} p^{m}, x_{j} \in\{0,1, \ldots, p-1\}$.

This is the basis of the number-theoretic representation of the space of social states.

In our model, $p$-adic balls represent social clusters corresponding to fixing a few social coordinates. For example $C_{j}=B_{1 / p}\left(a^{j}\right)=\{x \in$ $\left.\mathbf{Z}_{p}: x_{0}=j\right\}$, in above epidemic coding $C_{j}$ corresponds to fixing age $=$ $j ; C_{j i}=B_{1 / p}\left(a^{j i}\right)=\left\{x \in \mathbf{Z}_{p}: x_{0}=j, x_{1}=i\right\}$, age $=j$, gender $=i$ for Swedish society or age $=j$, income level $=i$ for American society.

Social states, points of $\mathbf{Q}_{p}$, can be considered as balls of zero radius, we call them elementary social clusters. Partitions of a ball into disjoint balls of smaller radii corresponds to partition of a social cluster into disjoint subclusters of deeper level of social hierarchy.

Now we turn to the algebraic representation of social states by rational numbers, see (7). The space $\mathbf{Q}_{p}$ endowed with ultrametric $\rho_{p}$ can be considered as completion of this set of rational numbers and algebraically the elements of $\mathbf{Q}_{p}$ can be represented by power series of the form

$$
x=\sum_{k=n} x_{k} p^{k}
$$

where $x_{j} \in\{0,1, \ldots, p-1\}, x_{n} \neq 0$, and $n \in \mathbf{Z}$; so only finite number of coordinates with negative index $k$ can differ from zero. 


\section{Disease spread as ultrametric diffu- sion equation on social landscapes}

An elementary social cluster (social state) given by a point of $\mathbf{Q}_{p}$ is the mathematical abstraction. Real clusters are represented by balls of finite radii. Therefore it is interesting to study the evolution of average probability for cluster $C \equiv B_{p^{n}}(0), n=0, \pm 1, \pm 2, \ldots$ For this purpose, a measure for averaging has to be selected. We point out that $\mathbf{Q}_{p}$ is an additive locally compact group. It can be endowed with shift-invariant measure, the Haar measure: for each ball, its measure equals to its radius. This is the simplest choice of averaging and we shall proceed with it. ${ }^{6}$ So, the average probability is represented as the integral with respect to the Haar measure:

$$
p_{I}(C, t)=\int_{C} p_{I}(x, t) \mu(d x) .
$$

The following master equation for probability $p_{I}(x, t)$ can be used

$$
\frac{\partial p_{I}(x, t)}{\partial t}=\int_{Q_{p}}\left[p(x \mid y ; t) p_{I}(y, t)-p(y \mid x ; t) p_{I}(x, t)\right] \mu(d y),
$$

where $p(x \mid y ; t)$ is the transition probability: the probability that the virus being present in (elementary) cluster $y$ would jump to cluster $x$. Once again, to simplify modeling it is supposed that this probability does not depend on time $t$ and it is symmetric, i.e., $p(x \mid y)=p(y \mid x)$. Under these assumptions, the master equation has the form

$$
\frac{\partial p_{I}(x, t)}{\partial t}=\int_{Q_{p}} p(x \mid y)\left[p_{I}(y, t)-p_{I}(x, t)\right] \mu(d y) .
$$

It is natural to assume that the transition probability decreases with increasing of the distance between two clusters, for example, that

$$
p(x \mid y)=\frac{C_{\alpha}}{|x-y|_{p}^{1+\alpha}}, \alpha>0 .
$$

Here $C_{\alpha}>0$ is a normalization constant, by mathematical reasons it is useful to select distance's power larger than one.

This function rather slowly approaches zero if the distance between points goes to infinity. Thus, the probability of transmission

\footnotetext{
${ }^{6}$ This choice, although so attractive mathematically, is not justified socially. However, our experience of ultrametric investigations showed that the mathematical properties of a model do not depend so much on selection of a measure on $\mathbf{Q}_{p}$, only computations can become more complicated.
} 
of infection from cluster $y$ to cluster $x$ for socially distant clusters is practically zero. This property of the model implies slower (than in the standard models of disease spread) approaching herd immunity: for a virus, it difficult to spread between socially distant clusters. We also remark that if the distance between points goes to zero, then the probability (in fact, its density) approaches infinity. This implies very rapid spread of infection in small social clusters. In contrast to the standard SIR-like models, in our model the probability of transmission of infection depends crucially on social distance.

Hence,

$$
\frac{\partial p_{I}(x, t)}{\partial t}=C_{\alpha} \int_{Q_{p}} \frac{p_{I}(y, t)-p_{I}(x, t)}{|x-y|_{p}^{1+\alpha}} \mu(d y) .
$$

The integral operator in the right-hand side is the operator of fractional derivative $D^{\alpha}$ (the Vladimirov operator), see [46]. Thus, the dynamics of the probability to become infected for those belonging to an elementary social cluster is described by the p-adic diffusion equation:

$$
\frac{\partial p_{I}(x, t)}{\partial t}=D^{\alpha} p_{I}(x, t)
$$

To formulate the Cauchy problem, we have to add some initial probability distribution. We select the uniform probability distribution concentrated on a single ball, initially infected social cluster $C$,

$$
p_{I}(x, 0)=\frac{1}{\mu(C)} \begin{cases}1, & x \in C \\ 0, & x \notin C\end{cases}
$$

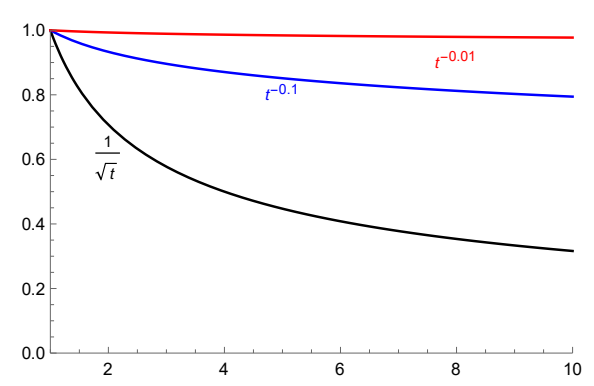

Figure 1: Asymptotic behavior of probability to become infected, transition probability parameter $\alpha=2,10,100$.

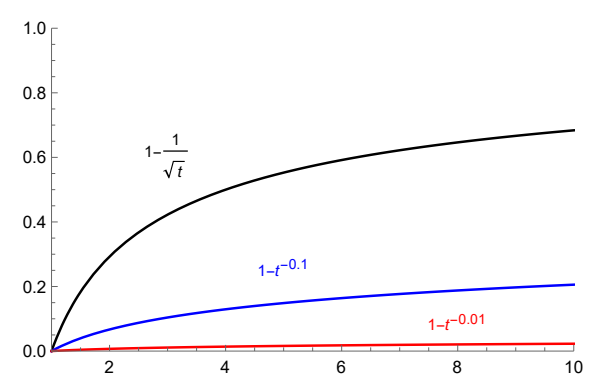

Figure 2: Asymptotic behavior of probability to become immune, transition probability parameter $\alpha=2,10,100$. 
This equation and its various generalizations were studied by many authors, for applications to physics and biology and by pure mathematical reasons [24]-[31].

We are interested in the relaxation regime, i.e., asymptotic of average probability $p_{I}(C, t)$ for large $t$. We use the mathematical result of above authors and obtain that the average probability has the power behavior:

$$
p_{I}(C, t) \sim t^{-1 / \alpha}, t \rightarrow \infty .
$$

Thus the average probability to become infected in a social cluster decreases rather slowly with time.

If parameter $\alpha$ is relatively large i.e., the virus transition probability decreases very quickly with increase of the distance between social clusters, then $p_{I}(C, t)$ decreases very slowly with time, it is practically constant (see the upper graph at Fig. 1).

If parameter $\alpha$ is relatively small, so the virus transition probability decreases slowly with increase of the distance, then $p_{I}(C, t)$ decreases sufficiently quickly with time (see the lowest graph at Fig. 1).

Consider now a kind of "integral immunity", combination of innate and adaptive components, defined as the probability of not become infected:

$$
p_{\operatorname{Im}}(x, t)=1-p_{I}(x, t)
$$

and its average over social cluster represented by ball $C$,

$$
p_{\operatorname{Im}}(C, t)=1-p_{I}(C, t)
$$

This function increases relatively slowly with time, see Fig. 2. Its asymptotic behavior depends on the parameter $\alpha$ determining how rapidly the transition probability between social clusters decreases with increase of the distance between them. The lowest graph corresponds to large value of $\alpha$, i.e., infection transition probability decreases very quickly. Then $p_{\operatorname{Im}}(C, t)$ is practically constant, herd immunity increases very slow.

Parameter $\alpha$ combines two different factors:

- Traditional social constraints in population.

- Preventing measures imposed by state authorities.

It is clear that existing of traditional rigid social barriers in population has similar effect as imposing of rigid preventing measures by authorities.

The parameter $\alpha$ can be represented as sum of two components, $\alpha=\alpha_{\text {soc }}+\alpha_{\text {preventing. }}$. For two populations (say countries) with large and small traditional social barriers $\alpha_{\mathrm{soc}}$, respectively, the same dynamics of herd immunity can be approached with small and large preventing barriers $\alpha_{\text {preventing, }}$, respectively. 
Say in Japan $\alpha_{\text {soc }}$ is relatively large and in Italy it is relatively small, so mild preventing measures in Japan would correspond to rigid preventing measures in Italy.

\section{Concluding remarks}

In physics, the ultrametric diffusion model can be considered as the continuous limit of random walk on trees representing energy landscapes. To move from one valley to another, a particle crosses barriers of different magnitude. In modeling the virus spread, social clusters play the role of valleys and barriers are the barriers between social clusters. The magnitudes of barriers depend on social, cultural, and historical traditions in population as well as on restrictions which were embossed by the state authorities during the covid-19 pandemic. Accounting the social clustering (see assumption AS0) and the specialty of infection's spreading (see assumption AS1) changes crucially the dynamics of infecting probability and consequently the immunity dynamics.

Our model implies that the strong restrictive measures during the covid-19 pandemic lead to the essential slowdown of increase of the herd immunity. As the result, no state in the world was able to approach the herd immunity.

This is a good example illustrating the importance of selection of an adequate mathematical model. State authorities followed recommendations of mathematicians who used inadequate models for covid19 spread. This policy made impossible the natural immunization of population and led to massive vaccination.

In this paper, we obtained only the asymptotic for solution of the ultrametric diffusion equation. This asymptotic is useful for study the problem of approaching the herd immunity. However, it would be even more interesting to obtain the exact analytic represenattion of solutions for arbitrary $t$. By using such solutions one can calculate the cumulative death rate. Preliminary numerical simulation which was done by the author shows that higher barriers imply (for the linear increase of barrier's magnitude) higher cummulative death rates.

This paper is based on the author's talk at. I would like to thank B. Dragovich and E. Zelenov for the discussion and fruitful recommendations. 


\section{References}

[1] A. Khrennikov, Ultrametric model for covid-19 dynamics: an attempt to explain slow approaching herd immunity in Sweden. https://www.preprints.org/manuscript/202007.0037/v1 ; https://www.medrxiv.org/content/10.1101/2020.07.04.20146209v1.

[2] A. Khrennikov and K. Oleschko, An ultrametric random walk model for disease spread taking into account social clustering of the population. Entropy 2020, 22(9), 931; https://doi.org/10.3390/e22090931 .

[3] R. M. Anderson, R. M. May, Infectious Diseases of Humans: Dynamics and Control (Oxford Univ. Press, 1991).

[4] H. Andersson, T. Britton, Stochastic Epidemic Models and Their Statistical Analysis (Springer, 2000).

[5] O. Diekmann, H. Heesterbeek, T. Britton, Mathematical Tools for Understanding Infectious Disease Dynamics (Princeton Univ. Press, 2013).

[6] D. Smith and L. Moore, The SIR Model for Spread of Disease - The Differential Equation Model. https://www.maa.org/press/periodicals/loci/joma/the-sirmodel-for-spread-of-disease-the-differential-equation-model.

[7] K. I. Kim, Zh. Lin, Q. Zhang, An SIR epidemic model with free boundary. Nonlinear Analysis: Real World Appl., 14, 2013, 1992-2001.

[8] S. Flaxman, S. Mishra, A. Gandy, H. J. T. Unwin, H. Coupland, T. A. Mellan, H. Zhu, T. Berah, J. W. Eaton, P. N. P. Guzman, N. Schmit, L. Cilloni, K. E. C. Ainslie, M. Baguelin, I. Blake, A. Boonyasiri, O. Boyd, L. Cattarino, C. Ciavarella, L. Cooper, Z. Cucunubá, G. Cuomo-Dannenburg, A. Dighe, B. Djaafara, I. Dorigatti, S. van Elsland, R. FitzJohn, H. Fu, K. Gaythorpe, L. Geidelberg, N. Grassly, W. Green, T. Hallett, A. Hamlet, W. Hinsley, B. Jeffrey, D. Jorgensen, E. Knock, D. Laydon, G. Nedjati-Gilani, P. Nouvellet, K. Parag, I. Siveroni, H. Thompson, R. Verity, E. Volz, C. Walters, H. Wang, Y. Wang, O. Watson, P. Winskill, X. Xi, C. Whittaker, P. G. T. Walker, A. Ghani, C. A. Donnelly, S. Riley, L. C. Okell, M. A. C. Vollmer, N. M. Ferguson, S. Bhatt, Report 13: Estimating the number of infections and the impact of non-pharmaceutical interventions on COVID-19 in 11 European countries (Imperial College London, 2020); .doi:10.25561/77731 
[9] N. M. Ferguson, D. Laydon, G. Nedjati-Gilani, N. Imai, K. Ainslie, M. Baguelin, S. Bhatia, A. Boonyasiri, Z. Cucunubá, G. Cuomo-Dannenburg, A. Dighe, I. Dorigatti, H. Fu, K. Gaythorpe, W. Green, A. Hamlet, W. Hinsley, L. C. Okell, S. van Elsland, H. Thompson, R. Verity, E. Volz, H. Wang, Y. Wang, P. G. T. Walker, C. Walters, P. Winskill, C. Whittaker, C. A. Donnelly, S. Riley, A. C. Ghani, Report 9: Impact of nonpharmaceutical interventions (NPIs) to reduce COVID-19 mortality and healthcare demand (Imperial College London, 2020); 10.25561/77482.doi

[10] T. Britton, Basic estimation-prediction techniques for Covid19, and a prediction for Stockholm. Preprint, April 2020 DOI: $10.1101 / 2020.04 .15 .20066050$

[11] T. Britton, Basic estimation-prediction techniques for Covid-19, and a prediction for Stockholm. https://www.medrxiv.org/content/10.1101/2020.04.15.20066050v2

[12] T. Britton, P. Trapman, F.G. Ball, The diseaseinduced herd immunity level for Covid-19 is substantially lower than the classical herd immunity level. https://www.medrxiv.org/content/10.1101/2020.05.06.20093336v2

[13] W. Bock, B. Adamik, M. Bawiec, V. Bezborodov, M. Bodych, J. P. Burgard, T. Goetz, T. Krueger, A. Migalska, B. Pabjan, T. Ozanski, E. Rafajlowicz, W. Rafajlowicz, E. SkubalskaRafajlowicz, S. Ryfczynska, E. Szczurek, P. Szymanski, Mitigation and herd immunity strategy for COVID-19 is likely to fail. medRxiv 2020.03.25.20043109 [Preprint]. 5 May 2020; .doi:10.1101/2020.03.25.20043109 .

[14] H. Salje, C. T. Kiem, N. Lefrancq, N. Courtejoie, P. Bosetti, J. Paireau, A. Andronico, N. Hozé, J. Richet, C.-L. Dubost, Y. Le Strat, J. Lessler, D. Levy-Bruhl, A. Fontanet, L. Opatowski, P.-Y. Boelle, S. Cauchemez, Estimating the burden of SARS-CoV-2 in France. Science 10.1126/science.abc3517 (2020). doi:10.1126/science.abc3517 .

[15] Khrennikov A., Human subconscious as a p-adic dynamical system. Journal of Theoretical Biology, 193(2), 179-96 (1998).

[16] D. Dubischar, V. M. Gundlach, O. Steinkamp, A. Khrennikov, A $p$-Adic Model for the Process of Thinking Disturbed by Physiological and Information Noise. Journal of Theoretical Biology 197(4), 451-67 (1999).

[17] A. Khrennikov, Information Dynamics in Cognitive, Psychological, Social, and Anomalous Phenomena. Kluwer, 2004 
[18] A. Khrennikov, Probabilistic pathway representation of cognitive information. J. Theor. Biology 231, N 4, 597-613 (2004).

[19] A. Khrennikov, Toward an adequate mathematical model of mental space: Conscious/unconscious dynamics on m-adic trees. Biosystems 90, N 3, 656-675 (2007).

[20] B. A. Huberman and M. Kerszberg, J. Phys. A 18, L331 (1985).

[21] Parisi, Phys. Rev. Lett. 43, 1754 (1979).

[22] Parisi, G.; Sourlas, N. p-Adic numbers and replica symmetry breaking. Eur. Phys. J. B 2000, 14, 535-542.

[23] A. T. Ogielski, Dynamics on Ultrametric Spaces. Phys. Rev. Lett, 55, 1634-1637.

[24] Kochubei, A. N.: Parabolic Equations over the Field of p-Adic Numbers, Math. USSR Izvestiya 39( 1992), 1263-1280

[25] S. Albeverio and X. Zhao, Measure-valued branching processes associated with random walks on p-adics. Ann. Probab. 28, (2000), 1680-1710.

[26] Kochubei, A.N. Pseudo-Differential Equations and Stochastics over Non-Archimedean Field; CRC Press: New York, NY, USA, 2001

[27] V. A. Avetisov, A. H. Bikulov, S. V. Kozyrev and V. A. Osipov, p-adic models of ultrametric diffusion constrained by hierarchical energy landscapes. J. Phys. A: Math. Gen. 35, 2002, 177-190.

[28] Kozyrev, S.V. Ultrametric dynamics as a model of interbasin kinetics. J. Comput. Math. Anal. 2006, 41, 38-48.

[29] Kozyrev, S.V. Ultrametric analysis and interbasin kinetics. AIP Conf. Proc. 2006, 826, 121-128.

[30] S. Albeverio, A. Yu Khrennikov, V. M. Shelkovich, Theory of P-adic Distributions: Linear and Nonlinear Models. Cambridge Univ. Press, Cambridge, 2010.

[31] Casas-Sanchez, O.F.; Zuniga-Galindo, W.A. P-adic elliptic quadratic forms, parabolic-type pseudodifferential equations with variable coefficients and Markov processes. P-Adic Numbers Ultrametr. Anal. Appl. 2014, 6, 120-139.

[32] T. Britton, F. Ball, P. Trapman, A mathematical model reveals the influence of population heterogeneity on herd immunity to SARS-CoV-2. Science 23 Jun 2020: eabc6810.

[33] J. Wallinga, P. Teunis, M. Kretzschmar , Using data on social contacts to estimate age-specific transmission parameters 
for respiratory-spread infectious agents. Am. J. Epidemiol. 164, 936-944 (2006).

[34] R. Pastor-Satorras, A. Vespignani, Epidemic spreading in scalefree networks. Phys. Rev. Lett. 86, 3200-3203 (2001).

[35] M. J. Ferrari, S. Bansal, L. A. Meyers, O. N. Bjørnstad, Network frailty and the geometry of herd immunity. Proc. Biol. Sci. 273, 2743-2748 (2006).

[36] J. Howard, Coronavirus spread by asymptomatic people appears to be rare," WHO official says. CNN, https://edition.cnn.com/2020/06/08/health/coronavirusasymptomatic-spread-who-bn/index.html

[37] A. Azad, CDC estimates that $35 \%$ of coronavirus patients don't have symptoms. CNN, May 22, 2020, https://edition.cnn.com/2020/05/22/health/cdc-coronavirusestimates-symptoms-deaths/index.html

[38] Technology Review, What's a coronavirus superspreader? https://www.technologyreview.com/2020/06/15/1003576/whatsa-coronavirus-superspreader/ 15.06.2020.

[39] Asano, M., Khrennikov, A., Ohya, M., Tanaka, Y. and Yamato, I. (2015). Quantum adaptivity in biology: from genetics to cognition, Springer: Heidelberg-Berlin-New York.

[40] Fuchs, C. A., Mermin, N. D. and Schack, R. (2014). An Introduction to QBism with an Application to the Locality of Quantum Mechanics, Am. J. Phys. 82, p. 749.

[41] Murtagh, F. The Haar wavelet transform of a dendrogram. J. Classif. 2007, 24, 3-32.

[42] Dragovich, B.; Dragovich, A. A p-Adic model of DNA sequence and genetic code. P-Adic Numbers Ultrametr. Anal. Appl. 2009, $1,34-41$.

[43] Dragovich, B.; Dragovich, A. p-adic modelling of the genome and the genetic code. Comput. J. 2010, 53, 432-442.

[44] Murtagh, F.; Contreras, P. Fast, linear time, m-adic hierarchical clustering for search and retrieval using the Baire metric, with linkages to generalized ultrametrics, hashing, formal concept analysis, and precision of data measurement. P-Adic Numbers Ultrametr. Anal. Appl. 2012, 4, 45-56.

[45] Contreras, P.; Murtagh, F. Fast, linear time hierarchical clustering using the Baire metric. J. Classif. 2012, 29, 118-143.

[46] Vladimirov, V.S.; Volovich, I.V.; Zelenov, E.I. P-Adic Analysis and Mathematical Physics; WSP: Singapore, 1994. 
[47] M. Mezard, G. Parisi, N. Sourlas, G. Toulouse, and M. Virasoro, Phys. Rev. Lett. 52, 1156 (1984).

[48] R. G. Palmer, Adv. Phys. 31, 66a (1982); G. Parisi, Phys. Rev. Lett. 50, 1946 (1983).

[49] A. Ansari, J. Berendzen, S. F. Bowne, H. Frauenfelder, I. E. T. Iben, T. B. Sauke, E. Shyamsunder, and R. D. Young, Proc. Natl. Acad. Sci. USA 82, 5000 (1985).

[50] R. G. Palmer, D. L. Stein, E. Abrahams, and P. W. Anderson, Phys. Rev. Lett. 53, 958 (1984).

[51] Public Health Institute of Sweden, Estimates of the peak-day and the number of infected individuals during the covid-19 outbreak in the Stockholm region, Sweden February-April 2020 [in Swedish] (2020); www.folkhalsomyndigheten.se/contentassets/2da059f90b90458d8454a04955d1697f/skattnin peakdag-antal-infekterade-covid-19-utbrottet-stockholms-lanfebruari-april-2020.pdf.

[52] Public Health Institute of Sweden: Antikroppstester avviker fran prognoserna - matematikern "ser tva förklaringar". https://www.svt.se/nyheter/inrikes/antikroppstester

[53] Public Health Institute of Sweden: Yngre personer har högre andel antikroppar. Sverige Radion, June 2, 20202; https://sverigesradio.se/sida/artikel.aspx?programid=83artikel $=7487102$

[54] Tegnell: "Vi får oroande rapporter om utelivet". SVT, Nyheter, 20-04-2020; https://www.svt.se/nyheter/inrikes/tegnellvi-far-oroande-rapporter-om-utelivet

[55] Se hur matematikprofessorn räknar ut när Stockholm uppnar flockimmunitet. SVT Nyheter, 20-04-2020.

[56] G. Holm, Hoppfulla teorin: Sa når vi flockimmunitet redan i juni. Expressen, 10.05.2020. https://www.expressen.se/nyheter/coronaviruset/hoppfullateorin-sa-nar-vi-flockimmunitet-redan-i-juni/

[57] A. Obminska, Det här vet vi om coronaviruset. $\mathrm{Ny}$ Teknik, 30-06-20202; https://www.nyteknik.se/samhalle/dethar-vet-vi-om-coronaviruset-6985117

[58] Public Health Institute of Sweden, 18-06-2020; https://www.folkhalsomyndigheten.se/nyheter-ochpress/nyhetsarkiv/2020/juni/forsta-resultaten-om-antikropparefter-genomgangen-covid-19-hos-blodgivare/ 
[59] L. A. Demetrius, V. M. Gundlach, Directionality Theory and the Entropic Principle of Natural Selection. Entropy 2014, 16, 5428-5522.

[60] Khrennikov, A. (2010). Ubiquitous quantum structure: from psychology to finances; Springer: Berlin-Heidelberg-New York.

[61] Busemeyer, J. R. and Bruza, P. D. (2012). Quantum models of cognition and decision, Cambridge University Press, Cambridge.

[62] Fuchs, C. A., Mermin, N. D. and Schack, R. (2014). An Introduction to QBism with an Application to the Locality of Quantum Mechanics, Am. J. Phys. 82, p. 749.

[63] H. Streeck, So far, no transmission of the virus in supermarkets, restaurants or hairdressers has been proved. https://today.rtl.lu/news/science-andenvironment/a/1498185.html, 14.04.2020.

[64] Podcast "Coronavirus-Update" mit Christian Drosten. https://www.ndr.de/nachrichten/info/14-Coronavirus-UpdateVorsicht-vor-Vereinfachungen,podcastcoronavirus132.html

[65] A. Khrennikov, Social Laser. Jenny Stanford Publ., Singapore, 2020.

[66] J. Goldstein, $68 \%$ Have antibodies in this clinic. Can a neighborhood beat a next wave? https://www.nytimes.com/2020/07/09/nyregion/nyccoronavirus-antibodies.html ; 9.07.2020.

[67] I. Garcia, Omradena som drabbats hardast av corona i Stockholm.

https://sverigesradio.se/sida/artikel.aspx?programid=83\&artikel $=7447621$ ; 7.04.2020.

[68] K Sato and M. Ohya, Evolution of HIV-1 from the viewpoint of information theory. 2010 3rd International Symposium on Applied Sciences in Biomedical and Communication Technologies (ISABEL 2010).

[69] M. Regoli, From Cryptography to Biology and Vice Versa. Open Systems and Information Dynamics 18, No. 01, 87-105 (2011). 